MT-DP - 2014/2

\title{
The kernel is in the least core for permutation games
}

TAMÁS SOLYMOSI 


\section{Discussion papers}

MT-DP - 2014/2

Institute of Economics, Centre for Economic and Regional Studies, Hungarian Academy of Sciences

KTI/IE Discussion Papers are circulated to promote discussion and provoque comments. Any references to discussion papers should clearly state that the paper is preliminary. Materials published in this series may subject to further publication.

The kernel is in the least core for permutation games

Author:

Tamás Solymosi associate professor Department of Operations Research and Actuarial Sciences Corvinus University of Budapest and 'Momentum' Game Theory Research Group, Institute of Economics, Research Center for Economic and Regional Studies, Hungarian Academy of Sciences

E-mail: tamas.solymosi@uni-corvinus.hu

January 2014

ISBN $978-615-5447-10-5$

ISSN $1785377 \mathrm{X}$ 


\title{
The kernel is in the least core for permutation games
}

\author{
Tamás Solymosi
}

\begin{abstract}
Permutation games are totally balanced transferable utility cooperative games arising from certain sequencing and re-assignment optimization problems. It is known that for permutation games the bargaining set and the core coincide, consequently, the kernel is a subset of the core. We prove that for permutation games the kernel is contained in the least core, even if the latter is a lower dimensional subset of the core. By means of a 5 -player permutation game we demonstrate that, in sense of the lexicographic center procedure leading to the nucleolus, this inclusion result can not be strengthened. Our 5-player permutation game is also an example (of minimum size) for a game with a non-convex kernel.
\end{abstract}

Keywords: permutation game, least core, kernel

JEL classification: C71

\section{Acknowledgements:}

Research supported by OTKA grant K-101224 and by the Hungarian Academy of Sciences under its Momentum Programme (LD-004/2010). The author is grateful to Holger Meinhardt and to two anonymous referees for their comments and suggestions on how to improve the presentation. 


\title{
Permutációs játékokban a kernel a szűkmag része
}

\author{
Solymosi Tamás
}

\section{Összefoglaló}

A permutációs játékok olyan teljesen kiegyensúlyozott kooperatív játékok, amelyek szorosan kapcsolódnak bizonyos ütemezési és sorbarendezési optimalizációs problémákhoz. Ismert, hogy a permutációs játékokban az alkuhalmaz és a mag egybeesnek, következésképpen a kernel a mag része. Megmutatjuk, hogy permutációs játékokban a kernel a szúkmagnak is része, még abban az esetben is, amikor a szúkmag a mag egy alacsonyabb dimenziós részhalmaza. Egy ötszereplős permutációs játék segítségével megmutatjuk, hogy ez a tartalmazási eredmény nem erősíthető a nukleoluszhoz vezető lexikografikus közép eljárás által generált egymásba ágyazott részhalmaz-sorozat további tagjaira. Ez az ötszereplős játék egyúttal egy minimális méretü példa arra is, hogy a kernel nem feltétlenül egy konvex halmaz.

Tárgyszavak: permutációs játék, szűkmag, kernel

JEL kód: C71 


\title{
The kernel is in the least core for permutation games *
}

\author{
TAMÁs Solymosi ${ }^{\dagger}$
}

December 15, 2013

\begin{abstract}
Permutation games are totally balanced transferable utility cooperative games arising from certain sequencing and re-assignment optimization problems. It is known that for permutation games the bargaining set and the core coincide, consequently, the kernel is a subset of the core. We prove that for permutation games the kernel is contained in the least core, even if the latter is a lower dimensional subset of the core. By means of a 5-player permutation game we demonstrate that, in sense of the lexicographic center procedure leading to the nucleolus, this inclusion result can not be strengthened. Our 5-player permutation game is also an example (of minimum size) for a game with a non-convex kernel.
\end{abstract}

JEL classification code: C71.

AMS 2010 classification. Primary: 91A12; Secondary: 91A40.

Keywords: permutation game, least core, kernel.

* Research supported by OTKA grant K-101224 and by the Hungarian Academy of Sciences under its Momentum Programme (LD-004/2010). The author is grateful to Holger Meinhardt and to two anonymous referees for their comments and suggestions on how to improve the presentation.

${ }^{\dagger}$ Department of Operations Research and Actuarial Sciences, Corvinus University of Budapest, H-1828 Budapest, Pf. 489, Hungary; and 'Momentum' Game Theory Research Group, Institute of Economics, Research Center for Economic and Regional Studies, Hungarian Academy of Sciences, H-1112, Budapest, Budaörsi út 45. E-mail: tamas.solymosi@uni-corvinus.hu. 


\section{Introduction}

Permutation games were introduced by Tijs, Parthasarathy, Potters and Rajendra Prasad (1984) in connection with certain sequencing and re-assignment problems. They proved that permutation games are totally balanced. Another proof of this was given by Curiel and Tijs (1986), who established relations between permutation games and assignment games, a class of games introduced by Shapley and Shubik (1972) in connection with certain two-sided matching situations. This connection was further explored by Quint (1996). He showed that the entire core of a permutation game can be obtained from the core of a related assignment game.

In this paper we focus on the kernel of permutation games. The kernel, introduced by Davis and Maschler (1965), is a nonempty set of imputations for all games with a nonempty imputation set. Both the kernel and the core are subsets of the bargaining set, a non-empty set-valued solution that was first investigated by Davis and Maschler (1967), but in general there is no inclusion relation between the kernel and the core. It was shown by Solymosi, Raghavan and Tijs (2003) that in permutation games the bargaining set coincides with the core, so we immediately get that in permutation games the kernel is a subset of the core.

In this paper we focus on the question whether in permutation games we can identify a subset of the non-empty core which contains the kernel. The first natural candidate is the least core, a non-empty set-valued solution that was formally introduced by Maschler, Peleg and Shapley (1979), since for balanced games it is a subset of the core. Our main result is that in permutation games the kernel is contained even in the least core.

The nucleolus-allocation, the single imputation in the nucleolus introduced by Schmeidler (1969), is always an element of both the kernel and the least core. It is the unique outcome of the so-called lexicographic center procedure, an optimization process first formalized by Maschler, Peleg and Shapley (1979), which sequentially reduces the set of allowable payoffs until it shrinks to a singleton. For balanced games, the core can be chosen as the initial set in this process, the first iteration reduces the allowable set to the least core, and finally, the lastly reduced allowable set contains only the nucleolus-allocation. Our main result can be rephrased as follows: in permutation games, the kernel is a subset of the allowable set determined by the first iteration of the lexicographic center procedure. By means of an example we demonstrate that in general this result cannot be sharpened. We give a 5player permutation game with a (non-convex) kernel that is not contained in the (convex) allowable set determined by the second iteration of the lexicographic center procedure, while the nucleolus-allocation is obtained only after the third iteration.

It was shown by Peleg (1966) that if the number of players in any game (with a non-empty imputations set) is at most four, the kernel is a convex set, in fact, a line segment or a singleton. In our 5-player permutation game the kernel is the union of two connected line segments with different direction vectors, so it is also 
an example (of the smallest possible size) for a game with a non-convex kernel. We remark that a $(5+5)$-player assignment game with a non-convex kernel was given by Granot and Granot (1992). The challenging task of computing the (pre)kernel in a general TU game is the topic of the recent book by Meinhardt (2014).

The organization of the paper is as follows. We recall the necessary definitions and preliminaries for general games in the next section. In section 3, we summarize the relevant known results for permutation games, highlight the subclass of cyclic permutation games and collect their known structural features. We show our main result and demonstrate that, in a certain sense, it can not be strengthened in section 4.

\section{Definitions and general preliminaries}

A transferable utility cooperative game on the nonempty finite set $N$ of players is defined by a coalitional function $V: 2^{N} \longrightarrow \mathbb{R}$ that satisfies $V(\emptyset)=0$. The function $V$ specifies the worth of every coalition $S \subseteq N$. We shall denote by

$$
\mathcal{P}:=\{S \subseteq N: S \neq \emptyset, N\}
$$

the collection of proper coalitions, and for distinct players $i$ and $j$, by

$$
\mathcal{P}_{i j}:=\{S \subseteq N: i \in S, j \notin S\}
$$

the collection of (proper) coalitions containing $i$ but not $j$.

A subcollection $\mathcal{S}$ of $\mathcal{P}$ is called balanced, if there are positive weights $\gamma_{S}, S \in \mathcal{S}$, such that $\sum_{S \in \mathcal{S}, S \ni i} \gamma_{S}=1$ for all $i \in N$. A balanced collection is said to be minimal, if it contains no proper balanced subcollection. It is well known (cf. Shapley, 1967) that a balanced collection is minimal if and only if the system of balancing weights is unique.

The game $(N, V)$ is called superadditive, if $S \cap T=\emptyset$ implies $V(S \cup T) \geq$ $V(S)+V(T)$ for all $S, T \subseteq N$; balanced, if $V(N) \geq \sum_{S \in \mathcal{S}} \gamma_{S} V(S)$ for every minimal balanced collection $\mathcal{S}$ with its unique system of weights $\gamma_{S}, S \in \mathcal{S}$; and totally balanced, if every subgame (i.e., the game obtained by restricting the player set to a coalition and the coalitional function to the power set of that coalition) is balanced. Note that totally balanced games are superadditive.

Given a game $(N, V)$, a payoff allocation $x \in \mathbb{R}^{N}$ is called efficient, if $x(N)=$ $V(N)$; individually rational, if $x_{i}=x(\{i\}) \geq V(\{i\})$ for all $i \in N$; coalitionally rational, if $x(S) \geq V(S)$ for all $S \subseteq N$; where, by the standard notation, $x(S)=$ $\sum_{i \in S} x_{i}$ if $S \neq \emptyset$, and $x(\emptyset)=0$. We denote by $\mathcal{X}^{*}(N, V)$ the preimputation set (i.e., the set of efficient payoff allocations), by $\mathcal{X}(N, V)$ the imputation set (i.e., the set of efficient and individually rational payoffs), and by $\mathcal{C}(N, V)$ the core (i.e., the set of efficient and coalitionally rational payoffs) of the game $(N, V)$. It is well known (cf. 
Shapley, 1967) that for transferable utility games balancedness and nonemptiness of the core are equivalent properties.

Given a game $(N, V)$, the excess $e(S, x):=V(S)-x(S)$ is the usual measure of gain (or loss if negative) to coalition $S \subseteq N$ if its members depart from allocation $x \in \mathbb{R}^{N}$ in order to form their own coalition. Note that $e(\emptyset, x)=0$ for all $x \in \mathbb{R}^{N}$, and

$$
\mathcal{C}(N, V)=\left\{x \in \mathbb{R}^{N}: e(N, x)=0, \quad e(S, x) \leq 0 \forall S \in \mathcal{P}\right\},
$$

i.e., the core is the set of efficient allocations which yield nonpositive excess for all coalitions.

The kernel, introduced by Davis and Maschler (1965), is a nonempty set of imputations for all games with a nonempty imputation set. It was proved by Maschler, Peleg and Shapley (1972) that for a large class of games (which includes superadditive games) the following alternative definition can be used. The kernel $\mathcal{K}(N, V)$ of the game $(N, V)$ is the set of all imputations $x$ satisfying

$$
s_{i j}(x)=s_{j i}(x) \quad \text { for all } i, j \in N, i \neq j,
$$

where for distinct players $i$ and $j$,

$$
s_{i j}(x):=\max _{S \in \mathcal{P}_{i j}} e(S, x)
$$

measures - in terms of excesses - the most $i$ can hope to gain (the least to lose) without the consent of $j$, if $i$ refuses allocation $x$. In this sense, the kernel (of superadditive games) consists of imputations which are in bilateral equilibrium for all pairs of players.

The nucleolus, an excess-based solution that is closely related to the kernel, was introduced by Schmeidler (1969), who proved that for games with a nonempty imputation set the nucleolus is always a nonempty subset of the kernel that consists of a single element. The following alternative definition of the nucleolus given by Maschler, Peleg and Shapley (1979) will serve us better here. Since we only deal with balanced games in this paper and for balanced games the nucleolus is in the core, we initiate the lexicographic center procedure with the core (instead of the imputation set).

Let $X^{0}:=\mathcal{C}(N, V)$ and $\Sigma^{0}:=\mathcal{P}$.

For $r=1, \ldots, \varrho$ define recursively

$$
\mid \begin{aligned}
& \varepsilon^{r}:=\min _{x \in X^{r-1}} \max _{S \in \Sigma^{r-1}} e(S, x), \\
& X^{r}:=\left\{x \in X^{r-1}: \max _{S \in \Sigma^{r-1}} e(S, x)=\varepsilon^{r}\right\}, \\
& \Sigma_{r}:=\left\{S \in \Sigma^{r-1}: \min _{x \in X^{r}} e(S, x)=\max _{x \in X^{r}} e(S, x)\right\}, \\
& \Sigma^{r}:=\Sigma^{r-1} \backslash \Sigma_{r},
\end{aligned}
$$

where $\varrho$ is the first value of $r$ for which $\Sigma^{r}=\emptyset$. 
The final set $X^{\varrho}$ is the nucleolus $\mathcal{N}(N, V)$ of the balanced game $(N, V)$. We refer to the unique vector in $X^{\varrho}$ as the nucleolus-allocation.

The least core $\mathcal{L C}(N, V)$ of a game $(N, V)$ was first formally treated by Maschler, Peleg and Shapley (1979) as the set of all efficient allocations that minimize the maximum excess of proper coalitions, i.e.,

$$
\mathcal{L C}(N, V):=\arg \min _{x \in \mathcal{X}^{*}(N, V)} \max _{S \in \mathcal{P}} e(S, x) .
$$

Obviously, for balanced games, the least core is exactly the set of optimal imputations in the first iteration of procedure (2), i.e., $\mathcal{L C}=X^{1} \subseteq \mathcal{C}$. Moreover, $\mathcal{L C}=\mathcal{C}$ if and only if $\varepsilon^{1}=0$. The following well-known characterization of least-core allocations will be used (cf. e.g. Peleg and Sudhölter, 2003, p.183):

Proposition 1 An efficient payoff allocation belongs to the least core if and only if the collection of proper coalitions having maximal excess at that allocation contains a (minimal) balanced collection.

Another combinatorial property of the collection of proper coalitions having maximum excess at an imputation appears in connection with the kernel (of superadditive games). We call a collection of proper coalitions $\mathcal{E} \subseteq \mathcal{P}$ separating, if for any two distinct players $i, j \in N$, whenever there is a coalition $S \in \mathcal{E}$ with $i \in S \not \supset j$ then there is a coalition $T \in \mathcal{E}$ with $j \in T \not \supset i$. By recalling the surplus-equalizing conditions (1) which define kernel elements, the following necessary condition is easily derived.

Proposition 2 If an imputation belongs to the kernel then the collection of proper coalitions having maximal excess at that imputation is a separating collection.

We note by passing that for a large class of games (which includes superadditive games), if the number of players is at most four then the kernel is contained in the least core. Indeed, in light of Propositions 1 and 2, it suffices to observe (cf. Solymosi, 2002, section 4) that for a player set $N$ with $|N| \leq 4$, any separating collection contains a (minimal) balanced collection. This is no longer true if $|N| \geq 5$ (for $|N|=5$ take, for example, the collection $\{134,135,145,234,235,245\}$ ), explaining why it is possible to construct (even totally) balanced games on a player set $N$ with $|N| \geq 5$ having kernel imputations outside the core. We must point out that these results were already (although implicitly) obtained by Peleg (1966), who proved that in 4-player games the kernel is a line segment (which may shrink into a single point), and that it occupies a central position in the core, whenever the core is not empty. It is easily checked that the line segment in Peleg's proof is precisely (what is nowadays called) the least core (and the single point the line segment may shrink into is the nucleolus). Peleg (1966) also presented a 5-player balanced game whose kernel is a line segment but has points outside the core.

Our aim in this paper is to show that on an interesting subclass of totally balanced games, the kernel is a subset of the least core, independently of the number of players. 


\section{$3 \quad$ Permutation games}

Given two finite sets $S$ and $T$, we call $\mu \subseteq S \times T$ an $(S, T)$-assignment, if it is a bijection from some $S^{\prime} \subseteq S$ to some $T^{\prime} \subseteq T$ such that $\left|S^{\prime}\right|=\left|T^{\prime}\right|=\min (|S|,|T|)$. Trivially, $\mu=\emptyset$ if $S=\emptyset$ or $T=\emptyset$. We shall write $(i, j) \in \mu$ as well as $\mu(i)=j$. We denote by $\Pi_{(S, T)}$ the set of all $(S, T)$-assignments. Obviously, $\Pi_{(S, T)}=\{\emptyset\}$ if $S=\emptyset$ or $T=\emptyset$. An $(S, S)$-assignment is called an $S$-permutation. We denote by $\Pi_{S}$ the set of all $S$-permutations. We call an $S$-permutation $\sigma$ cyclic, if for any proper subset $S^{\prime}$ of $S$, the restriction of $\sigma$ to $S^{\prime}$ is not an $S^{\prime}$-permutation. A cycle of an $S$-permutation $\sigma$ is the restriction of $\sigma$ to a subset $S^{\prime}$ of $S$, if the restriction is a cyclic $S^{\prime}$-permutation. Clearly, every permutation is the union of its cycles.

A game $(N, V)$ is called a permutation game if there exists a square matrix $A=\left[a_{i j}\right]_{i \in N, j \in N}$ such that

$$
V(S)=\max _{\sigma \in \Pi_{S}} \sum_{i \in S} a_{i \sigma(i)} \quad \forall S \subseteq N
$$

Note that $\Pi_{S}$ is nonempty and finite for all $S \subseteq N$, so $V$ is well defined. Moreover, $V(\emptyset)=0$. Naturally, many different matrices induce the same game. Given a fixed matrix $A$, we denote by $\Pi_{S}^{*}$ the set of optimal solutions to (3). An element of $\Pi_{S}^{*}$, typically denoted by $\sigma_{S}$, will be called a maximal $S$-permutation in the matrix $A$. Note that permutation games are superadditive.

The (total) balancedness of permutation games was first proved by Tijs et al. (1984). We briefly summarize the alternative proof given by Curiel and Tijs (1986), because it leads to a useful representation of core allocations. Let $(N, V)$ be the permutation game induced by the matrix $A$, and let $\pi$ be a fixed maximal $N$ permutation, i.e., $V(N)=\sum_{i \in N} a_{i \pi(i)}$. Without indicating the dependence on this fixed $\pi$, we simply denote by $H:=N \times N \backslash \pi$ the set of off- $\pi$ pairs of players.

It is well-known that $\pi$ can be obtained by solving a linear programming problem. We denote the set of optimal dual solutions to this LP by

$$
\mathcal{D}=\left\{(u, v) \in \mathbb{R}^{N+N}: d_{i j}(u, v)=0 \forall(i, j) \in \pi, d_{i j}(u, v) \leq 0 \forall(i, j) \in H\right\},
$$

where $d_{i j}(u, v):=a_{i j}-u_{i}-v_{j}$ denotes the slack of the $(i, j) \in N \times N$ dual constraint at dual vector $(u, v) \in \mathbb{R}^{N} \times \mathbb{R}^{N}$. Let the mapping $m: \mathbb{R}^{N} \times \mathbb{R}^{N} \longrightarrow \mathbb{R}^{N}$ be defined by $m(u, v):=u+v$. Curiel and Tijs $(1986)$ showed that $m(\mathcal{D}) \subseteq \mathcal{C}$, consequently $\mathcal{C}$ is nonempty. The reverse inclusion $m(\mathcal{D}) \supseteq \mathcal{C}$ was proved by Quint (1996). The representation

$$
\mathcal{C}(N, V)=m(\mathcal{D})
$$

of the core will be fundamental for our investigation, since we can rephrase conditions imposed on core allocations of the permutation game in terms of optimal dual vectors related to the underlying matrix.

As a consequence of the balancedness of permutation games and two other known results, we get the following inclusion statement. 
Proposition 3 In a permutation game, the kernel is a subset of the core.

Indeed, the inclusion immediately follows from two known results: first, the kernel is always a subset of the bargaining set (Davis and Maschler, 1965); second, in permutation games the bargaining set coincides with the core (Solymosi et al., 2003).

In this paper, our aim is to show the stronger statement that in permutation games, the kernel is included even in the least core. Obviously, there is nothing to prove, if the grand coalition is inessential in the game (i.e. its value is weakly majorized by the value of one of its proper partitions), for in such cases the least core coincides with the core. Assignment games are prime examples for permutation games where the grand coalition is inessential. We remark that for assignment games the inclusion of the kernel in the (least) core follows from the work of Granot (1994) and Granot (2010), whereas Driessen (1998) proves the inclusion directly.

In the rest of this section we consider an important subclass of permutation games, called cyclic permutation games. It contains all permutation games in which the least core is a proper subset of the core. In particular, it contains permutation games in which the grand coalition is essential (i.e., $V(N)>V\left(N_{1}\right)+\ldots+V\left(N_{k}\right)$ for every proper partition $N=N_{1} \cup \ldots \cup N_{k}, k \geq 2$, of coalition $N$ ). On the other hand, assignment games are also cyclic permutation games, although in them most of the multi-player coalitions (including the grand coalition) are inessential. This contrast implies that the structural features of cyclic permutation games are the key factors for our main result and not the essentiality of the grand coalition or the full dimensionality of the core.

We call a permutation game $(N, V)$ cyclic, if it is induced by a cyclic matrix $A$, i.e. if there exists a cyclic $N$-permutation which is of maximum value in $A$. In order to simplify the notation, from now on we shall assume that $N=\{1, \ldots, n\}$, and the cyclic $N$-permutation

$$
\pi(i):=\left\{\begin{array}{cl}
i+1 & \text { if } i \neq n \\
1 & \text { if } i=n
\end{array}\right.
$$

is of maximum value in the underlying matrix $A$, i.e., $V(N)=\sum_{i \in N} a_{i \pi(i)}$.

Given the fixed cyclic permutation $\pi$, for $i, j \in N$ we define the interval coalition from $i$ to $j$ as

$$
[i, j]:= \begin{cases}\left\{i, \pi(i), \ldots, \pi^{k}(i)=j\right\} & \text { if } i \neq j \text { and } 1 \leq k \leq n-1 \\ \{i\} & \text { if } i=j\end{cases}
$$

For example, $[1,2]=\{1,2\}$ because $\pi(1)=2$, but $[2,1]=N$ because $\pi(2)=$ $3, \ldots, \pi^{n-1}(2)=1$. It is clear that any coalition has a unique interval-partition consisting of its interval-components (i.e. the maximal-for-inclusion subcoalitions which are intervals).

The pair $(i, j)$ of players can be augmented with pairs in $\pi$ to form a cyclic permutation for the interval coalition $[j, i]$. Indeed, the mapping

$$
\sigma_{i j}(i):=j, \quad \text { and } \quad \sigma_{i j}(h):=\pi(h) \forall h \in[j, i] \backslash\{i\}
$$


is a cyclic $[j, i]$-permutation. Observe that $(i, j)$ is an off- $\pi$ pair if and only if the associated interval $[j, i]$ is a proper coalition. Let

$$
\mathcal{H}=\{[j, i]:(i, j) \in H\}
$$

denote the set of proper intervals. Since for $[j, i] \in \mathcal{H}$ the associated $\sigma_{i j}$ may not be of maximum value, we clearly have

$$
e([j, i], u+v) \geq \sum_{(p, q) \in \sigma_{i j}} d_{p q}(u, v)=d_{i j}(u, v) \quad \forall(u, v) \in \mathcal{D}
$$

so at any core allocation $x=u+v \in \mathcal{C}$ the excess of an interval coalition is not less than the associated dual slack at any corresponding optimal dual vector $(u, v) \in m^{-1}(x) \subseteq \mathcal{D}$. Let

$$
\mathcal{H}^{*}=\left\{[j, i] \in \mathcal{H}: \sigma_{i j} \in \Pi_{[j, i]}^{*}\right\} .
$$

denote the set of proper intervals for which the associated permutation is of maximal value, hence the weak inequality in (4) holds with equality. Notice that $[i, i] \in \mathcal{H}^{*}$ for all $i \in N$, i.e. all single-player coalitions belong to this subclass of intervals.

In the following lemma we summarize the key structural feaures of cyclic permutation games. The proofs and more details can be found in (Solymosi et al., 2005). We say that a collection $\mathcal{T} \subseteq \mathcal{H}$ reproduces $S \in \mathcal{P}$ (modulo $N$ ) if every player in $S$ is contained in exactly one more interval from $\mathcal{T}$ than any player not in $S$.

Lemma 1 (Solymosi, Raghavan, Tijs, 2005) In a cyclic permutation game, for any $S \in \mathcal{P}$ there is a collection $\mathcal{S} \subseteq \mathcal{H}^{*}$ which reproduces $S$ (modulo $N$ ), and a constant $t_{\mathcal{S}} \geq 0$ such that

$$
e(S, x)=\sum_{T \in \mathcal{S}} e(T, x)-t_{\mathcal{S}} \quad \forall x \in \mathcal{C} .
$$

Consequently,

$$
\max _{S \in \mathcal{P}} e(S, u+v)=\max _{T \in \mathcal{H}^{*}} e(T, u+v)=\max _{(p, q) \in H} d_{p q}(u, v) \quad \forall(u, v) \in \mathcal{D} .
$$

In light of Proposition 3, Lemma 1 implies that during the determination of core allocations based on maximum excesses we can ignore coalitions which are not intervals, because at core allocations the excess of any proper coalition is majorized by the excess of at least one interval coalition from the collection $\mathcal{H}^{*}$ (in fact, it is less than or equal to the excess of all the interval coalitions in the $\mathcal{H}^{*}$-decomposition of the coalition). Notice that this is a significant simplification possibility, since there are (in the number of players) exponential many proper coalitions, but only quadratic many of them are intervals. 


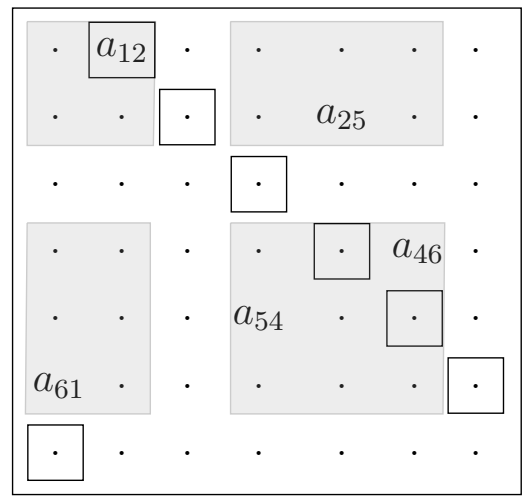

$$
\begin{aligned}
& (1,2) \leftrightarrow[2,1]=\begin{array}{lllllll}
1 & 2 & 3 & 4 & 5 & 6 & 7
\end{array} \\
& (2,5) \leftrightarrow[5,2]=12 \cdot \quad \cdot \quad 567 \\
& (4,6) \leftrightarrow[6,4]=\begin{array}{lllllll}
1 & 2 & 3 & 4 & \cdot & 6 & 7
\end{array} \\
& (5,4) \leftrightarrow[4,5]=\cdot \cdot \cdot 45 \text {. } \\
& (6,1) \leftrightarrow[1,6]=\begin{array}{lllllll}
1 & 2 & 3 & 4 & 5 & 6
\end{array} .
\end{aligned}
$$

Figure 1: Any permutation induces a reproducing family of intervals

As an illustrative example, consider the cyclic matrix in Figure 1 and the induced cyclic permutation game on player set $N=\{1, \ldots, 7\}$. The maximal $N$-permutation $\pi$ is indicated by the framed squares. Coalition $S=\{1,2,4,5,6\}$ is not an interval (its interval-components are $[1,2]$ and $[4,6]$ ), the corresponding principal submatrix is highlighted in light gray. Take the (cyclic) $S$-permutation $\sigma=\{(1,2),(2,5)$, $(5,4),(4,6),(6,1)\}$. The collection of the related intervals (listed in the reverse order) $\mathcal{S}_{\sigma}=\{[1,6],[6,4],[4,5],[5,2],[2,1]\}$ reproduces $S$ (modulo $N$ ) since each player in $S$ appears in exactly four, while each player in $N \backslash S$ in only three of these intervals. Both numbers decrease by one if we omit $[2,1]=N$ related to the only in$\pi$ pair $(1,2) \in \sigma$. Suppose the proper intervals $[1,6],[6,4],[4,5] \in \mathcal{S}_{\sigma}$ belong to $\mathcal{H}^{*}$, but for the proper interval $[5,2] \in \mathcal{S}_{\sigma}$ the value of permutation $\tau=\{(1,2),(2,6)$, $(6,1),(5,7),(7,5)\}$ is $t_{(2,5)} \geq 0$ higher than the value of its associated permutation $\sigma_{(2,5)}=\{(2,5),(5,6),(6,7),(7,1),(1,2)\}$. The collection of intervals related to $\tau$ (listed in the reverse order) $\mathcal{T}_{\tau}=\{[5,7],[7,5],[1,6],[6,2],[2,1]\}$ contains each player in $[5,2]$ four times, but each player in $N \backslash[5,2]=[3,4]$ only three times. Again, both numbers decrease by one if we omit $[2,1]=N$ related to the in- $\pi$ pair $(1,2) \in \tau$. Now, if in $\mathcal{S}_{\sigma}$ we replace $[5,2]$ with its reproducing family $\mathcal{T}_{\tau}$ and keep both copies of $[1,6]$ and $[2,1]=N$ related to the common elements $(6,1)$ and $(1,2) \in \sigma \cap \tau$, respectively, then we get a collection $\mathcal{S}^{\prime}=\mathcal{S}_{\sigma} \cup \mathcal{T}_{\tau} \backslash\{[5,2]\}$ in which each player in $S$ is listed exactly seven times, while each player in $N \backslash S$ only six times. Both numbers decrease by two if we omit the two copies of $[2,1]=N$ related to the common in- $\pi$ pair $(1,2)$. Thus, $\mathcal{S}=\mathcal{S}^{\prime} \cap \mathcal{H}$ also reproduces $S$ (modulo $N$ ). In case the above $\sigma$ is a maximal $S$-permutation and all (proper) intervals in $\mathcal{S}$ belong to $\mathcal{H}^{*}$, we have $e(S, x)=\sum_{T \in \mathcal{S}} e(T, x)-t_{(2,5)}$ for any $x \in \mathcal{C}$. It follows that for any $(u, v) \in m^{-1}(x) \subseteq \mathcal{D}$ we have $e(S, x) \leq d_{(p, q)}(u, v)$ for all $(p, q) \in(\sigma \cup \tau) \cap H$.

Before we can prove our main result, we also need to recall another feature of cyclic permutation games. In the above lemma we considered intervals related to $S$-permutations, i.e. to $(S, S)$-assignments. Now we look at intervals arising from $(S, \pi(S))$-assignments. A sequence of an even number of distinct pairs of players 
is said to be a loop, if it is of the form $\left[\left(i_{1}, j_{1}\right),\left(i_{1}, j_{2}\right),\left(i_{2}, j_{2}\right),\left(i_{2}, j_{3}\right), \ldots,\left(i_{k}, j_{k}\right)\right.$, $\left.\left(i_{k}, j_{k+1}\right)\right]$ such that $k \geq 2, j_{k+1}=j_{1}$ and $\pi\left(i_{h}\right)=j_{h+1} \forall h=1, \ldots, k$. Since every second pair must be in $\pi$, the sequence of the off- $\pi$ pairs $\left[\left(i_{h}, j_{h}\right): h=1, \ldots, k\right]$ completely determines the whole loop. (Notice that this sequence defines a bijection from $I=\left\{i_{1}, \ldots, i_{k}\right\}$ to $\pi(I)=\left\{j_{1}, \ldots, j_{k}\right\}$ disjoint from $\pi$.) In other words, a set of entries in the underlying matrix forms a loop if and only if each row and each column contains exactly zero or two entries from this set, and in the latter case one entry is from $\pi$.

We call a minimal balanced collection homogeneous if the (unique) balancing weights are all equal.

Lemma 2 (Solymosi, Raghavan, Tijs, 2005) In a cyclic permutation game, if the sequence $\left[\left(i_{h}, j_{h}\right): h=1, \ldots, k\right]$ of off- $\pi$ pairs generates a loop, the collection $\left\{\left[j_{h}, i_{h}\right]: h=1, \ldots, k\right\}$ of intervals is a homogeneous minimal balanced collection.

For illustration, take the cyclic $7 \times 7$-matrix in Figure 2 . Let $[(2,1),(6,3)$,

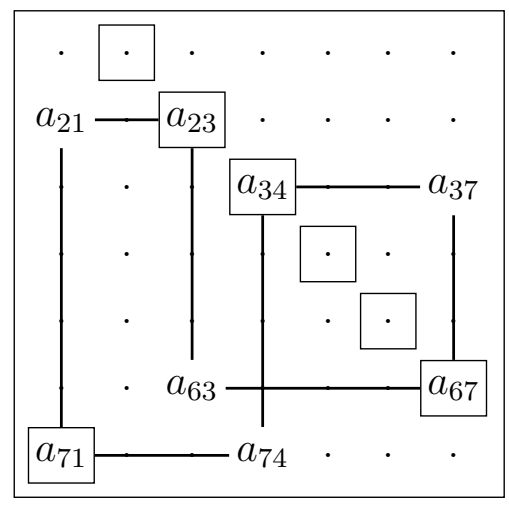

$$
\begin{aligned}
& (2,1) \leftrightarrow[1,2]=\begin{array}{lllll}
1 & 2 & . & . & .
\end{array} \\
& (3,4) \leftrightarrow[4,3]=\begin{array}{llllll}
1 & 2 & 3 & \cdot & \cdot &
\end{array} \\
& (6,3) \leftrightarrow[3,6]=\cdot .34 \quad 56 . \\
& (7,4) \leftrightarrow[4,7]=\cdot \cdot \cdot 4 \quad 5667
\end{aligned}
$$

Figure 2: A loop induces a minimal balanced family of intervals

$(3,7),(7,4)]$ be a sequence of off- $\pi$ pairs that determines the loop $[(2,1),(2,3)$, $(6,3),(6,7),(3,7),(3,4),(7,4),(7,1)]$. Then the collection of the corresponding proper intervals $\{[1,2],[3,6],[7,3],[4,7]\}$ covers every player exactly twice, so it can be balanced with equal $1 / 2$ weights.

\section{The main result}

As stated in Proposition 3, for all permutation games the kernel $\mathcal{K}$ is contained in the core $\mathcal{C}$. In this section we show that for all permutation games the kernel is contained even in the least core $\mathcal{L C}(\subseteq \mathcal{C})$. Of course, this claim needs explanation only when $\mathcal{L C} \neq \mathcal{C}$, i.e., when $\varepsilon^{1}<0$ in the lexicographic center procedure (2). This cannot happen if the game is not cyclic (i.e., no cyclic $N$-permutation is maximal), because then the cycles of a maximal $N$-permutation induce a proper partition 
$N=N_{1} \cup \ldots \cup N_{k}, k \geq 2$, of the player set such that $V(N)=V\left(N_{1}\right)+\ldots+V\left(N_{k}\right)$. Therefore, we restrict our discussion to cyclic permutation games. Notice that even in the cyclic case, $\varepsilon^{1}<0$ if and only if the maximal $N$-permutation is unique.

For distinct players $i$ and $j$, we introduce the set of (off- $\pi$ ) pairs

$$
H_{i j}:=\left[i, \pi^{-1}(j)\right] \times[\pi(j), i],
$$

and the collection

$$
\mathcal{H}_{i j}:=\mathcal{H} \cap \mathcal{P}_{i j}
$$

of interval coalitions which contain $i$ but not $j$. Clearly, $(p, q) \in H_{i j}$ if and only if $[q, p] \in \mathcal{H}_{i j}$. Figure 3 highlights the submatrices of off- $\pi$ pairs in a cyclic $(5 \times 5)$ matrix that induce the intervals which separate 1 from 4 (on the left) and those which separate 4 from 1 (on the right).

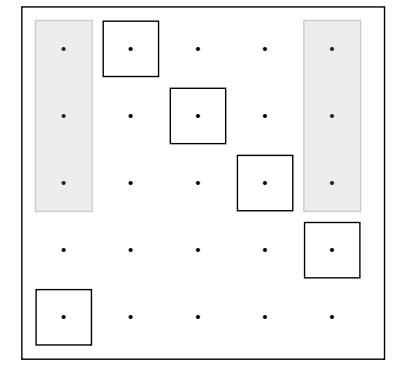

$H_{14}=[1,3] \times[5,1]$

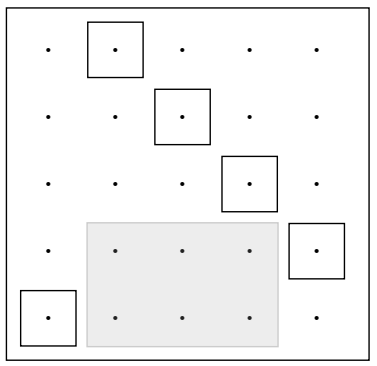

$H_{41}=[4,5] \times[2,4]$

Figure 3: Pairs induce intervals separating 1 and 4

We know from Lemma 1 that when in the core only maximum excess matters we can ignore coalitions which are not intervals. Next we show that the same is true for maximum excess proper coalitions which separate one player from another.

Lemma 3 In a cyclic permutation game, for all $i \neq j$ and $(u, v) \in \mathcal{D}$,

$$
s_{i j}(u+v)=\max _{T \in \mathcal{H}_{i j}} e(T, u+v)=\max _{(p, q) \in H_{i j}} d_{p q}(u, v) .
$$

In particular, $s_{i \pi(i)}(u+v)$ equals the maximum of the off- $\pi$ entries in row $i$ of the matrix $\left[d_{p q}(u, v)\right]$ of dual slacks at $(u, v) \in \mathcal{D}$; and $s_{\pi(i) i}(u+v)$ equals the maximum of the off- $\pi$ entries in column $\pi(i)$ of that matrix.

Proof Let $i \neq j$ and $(u, v) \in \mathcal{D}$.

Take an $S \in \mathcal{P}_{i j}$ such that $s_{i j}(u+v)=e(S, u+v)$, and a maximal $S$-permutation $\sigma_{S} \in \Pi_{S}^{*}$. It follows from

$$
e(S, u+v)=\sum_{p \in S} d_{p, \sigma_{S}(p)}(u, v)=\sum_{(p, q) \in \sigma_{S} \backslash \pi} d_{p q}(u, v)
$$


that $e(S, u+v) \leq d_{p q}(u, v) \leq 0$ for all $(p, q) \in \sigma_{S} \backslash \pi$ at the dual optimal vector $(u, v) \in \mathcal{D}$. Since $S$ contains $i$ but not $j$, Lemma 1 implies that at least one of the proper intervals in the reproducing family $[q, p],(p, q) \in \sigma_{S} \backslash \pi$, belongs to $\mathcal{H}_{i j}$, so at least one of the pairs $(p, q) \in \sigma_{S} \backslash \pi$ belongs to $H_{i j}$. Thus, $e(S, u+v) \leq d_{p q}(u, v)$ for some $(p, q) \in H_{i j}$. Therefore,

$$
s_{i j}(u+v)=e(S, u+v) \leq \max _{(p, q) \in H_{i j}} d_{p q}(u, v) \leq \max _{T \in \mathcal{H}_{i j}} e(T, u+v) \leq s_{i j}(u+v),
$$

where the second inequality comes from (4), and the third from $\mathcal{H}_{i j} \subseteq \mathcal{P}_{i j}$.

The claims for the special case $j=\pi(i)$ in (7) follow immediately from $H_{i \pi(i)}=$ $[i, i] \times\left[\pi^{2}(i), i\right]$ and $H_{\pi(i) i}=\left[\pi(i), \pi^{-1}(i)\right] \times[\pi(i), \pi(i)]$, respectively.

Now we are ready to prove our main result.

Theorem 1 If $(N, V)$ is a permutation game then $\mathcal{K}(N, V) \subseteq \mathcal{L C}(N, V)$.

Proof As remarked earlier, it suffices to show the inclusion in the cyclic case.

Let the cyclic permutation game $(N, V)$ be induced by the cyclic matrix $A$. Let us choose an $x \in \mathcal{K}$ arbitrarily. It follows from $\mathcal{K} \subseteq \mathcal{C}=m(\mathcal{D})$ that for all $(u, v) \in m^{-1}(x)$ we have $(u, v) \in \mathcal{D}$. So, let us choose an $(u, v) \in \mathcal{D}$ satisfying $x=u+v$ arbitrarily.

Given this fixed $(u, v) \in m^{-1}(x)$, we define $d(u, v):=\max _{(p, q) \in H} d_{p q}(u, v)$ and $M(u, v):=\arg \max _{(p, q) \in H} d_{p q}(u, v)$. If $(i, j) \in M(u, v)$, we get from Lemma 3 that $s_{i \pi(i)}(u+v)=d(u, v)$, since the maximum of the off- $\pi$ entries in row $i$ of the matrix $\left[d_{p q}(u, v)\right]$ of dual slacks at $(u, v)$ is $d(u, v)$. Then, $u+v \in \mathcal{K}$ implies that $s_{\pi(i) i}(u+v)=d(u, v)$ is the maximum of the off- $\pi$ entries also in column $\pi(i)$. If $\left(i^{\prime}, \pi(i)\right) \in M(u, v)$, then $i^{\prime} \neq i$ and, as before, we get that $d(u, v)$ is the maximum of the off- $\pi$ entries also in column $\pi\left(i^{\prime}\right) \neq \pi(i)$. Let $\left(i^{\prime \prime}, \pi\left(i^{\prime}\right)\right) \in M(u, v)$. If $i^{\prime \prime}=i$ then the sequence $\left[\left(i, \pi\left(i^{\prime}\right)\right),\left(i^{\prime}, \pi(i)\right)\right]$ of off- $\pi$ pairs generates a loop. (Note that $\pi\left(i^{\prime}\right)$ might be distinct from the starting $j$.) If $i^{\prime \prime} \neq i$ then we can iterate the above argument and get a new row and the corresponding column, both distinct from the previously taken rows and columns, respectively. Obviously, we eventually close a loop by getting an already considered row (that need not be the starting one). We conclude that $M(u, v)$ contains a sequence of off- $\pi$ pairs which generates a loop. By Lemma 2 and equation (6), this means that the collection of maximal excess coalitions at $x=u+v$ contains a (minimal) balanced collection. It follows from the characterization of least-core allocations stated in Proposition 1 that $x \in \mathcal{L C}$.

We illustrate the loop finding argument in the above proof with the help of Figure 4. In the matrices we indicated only the relevant dual slacks: $d$ is the maximum slack of the off- $\pi$ entries, and, of course, 0 is the constant slack for the in- $\pi$ entries. The matrix on the left shows a situation when the process starts with $(i, j)=(3,2)$, continues with $\left(i^{\prime}, \pi(i)\right)=(4,4)$, then with $\left(i^{\prime \prime}, \pi\left(i^{\prime}\right)\right)=(1,5)$, 

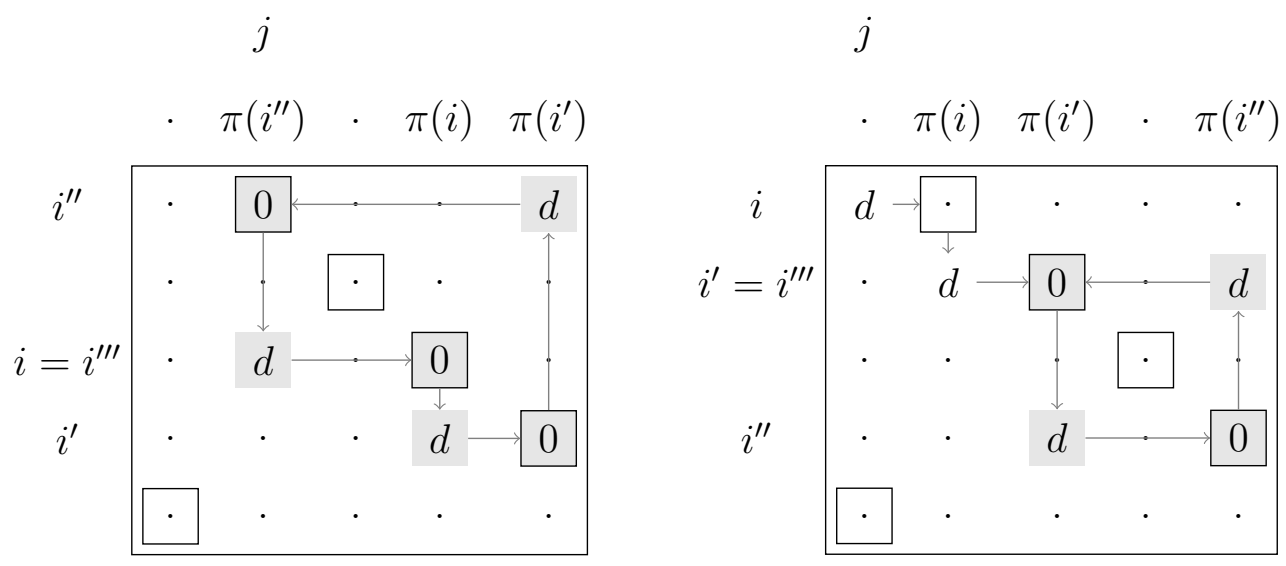

Figure 4: Both (left) or neither (right) starting row / column in loop

and terminates with $\left(i^{\prime \prime \prime}, \pi\left(i^{\prime \prime}\right)\right)=(3,2)$, the very entry we started with. In this case, both the initial row and column are covered by the loop found (highlighted in gray). In contrast, the matrix on the right shows a situation where neither the initial row nor the initial column is covered by the loop found. There we start from $(i, j)=(1,1)$, then find $\left(i^{\prime}, \pi(i)\right)=(2,2)$, then continue in the column $\pi\left(i^{\prime}\right)=3$ with $\left(i^{\prime \prime}, \pi\left(i^{\prime}\right)\right)=(4,3)$, and stop with $\left(i^{\prime \prime \prime}, \pi\left(i^{\prime \prime}\right)\right)=(2,5)$ because we reached the already covered row $i^{\prime}=2$. The loop we found is generated only by the last two off- $\pi$ entries discovered during the process.

Note that during the loop finding process applied in the above proof only the $s_{i \pi(i)}()=.s_{\pi(i) i}(),. i \in N$, kernel conditions were used. This prompts the question whether the $\mathcal{K} \subseteq X^{1}$ inclusion can be strengthened (maybe to the set $X^{2}$ or even further in the lexicographic center procedure (2)) by taking into consideration the surplus equalizing conditions also for the pairs not in $\pi$. In the rest of this section we demonstrate that the answer is no. In Example 1 below, we present a 5-player (cyclic) permutation game where $X^{1} \supset \mathcal{K} \supset X^{2} \supset \mathcal{N}$ (all inclusions are strict), implying that Theorem 1 can not be sharpened in sense of the lexicographic center procedure. The kernel is not a convex set in this 5-player game, so we also give a minimum size example for a game with a non-convex kernel, since the kernel is known to be convex for any general game with at most four players (and a nonempty imputation set) (Peleg, 1966, Lemma 3.1).

Example 1 The $5 \times 5$ cyclic matrix

$$
A=\left[\begin{array}{ccccc}
1 & 7 & 0 & 6 & 0 \\
1 & 0 & 2 & 0 & 1 \\
2 & 6 & 0 & 7 & 0 \\
0 & 0 & 1 & 0 & 2 \\
5 & 3 & 2 & 4 & 0
\end{array}\right]
$$


induces a 5-player cyclic permutation game for which $\mathcal{C}=X^{0} \supset \mathcal{L C}=X^{1} \supset \mathcal{K} \supset$ $X^{2} \supset \mathcal{N}$ (all inclusions are strict). Furthermore, $\mathcal{K}$ is not convex.

Since the row maximums (boxed) form a cyclic permutation of the players, the induced permutation game is indeed cyclic, so the non-interval coalitions can be ignored both in computing the surplus values for the kernel conditions and in the lexicographic center procedure.

Allocation $x=(7,2,7,2,5)$ is in the core and provides an excess of at most -1 for all coalitions and exactly -1 excess for the intervals shown on the right in Figure 5. On the left, the matrix of dual slacks at a particular $\left(u_{x}, v_{x}\right) \in m^{-1}(x)$

\begin{tabular}{|c|c|c|c|c|c|c|c|c|c|c|c|c|}
\hline$u_{x} \backslash v_{x}$ & 0 & 0 & 0 & 0 & 0 & $x$ & $=$ & 7 & 2 & 72 & 5 & $\in \mathcal{K} \cap X^{1}$ \\
\hline 7 & -6 & 0 & -7 & -1 & -7 & $(1,4)$ & $\leftrightarrow$ & 1 & . & . 4 & 5 & \multirow{6}{*}{$\varepsilon^{1}=-1$} \\
\hline 2 & -1 & -2 & 0 & -2 & -1 & $(3,2)$ & $\leftrightarrow$ & . & 2 & 3 & . & \\
\hline & & & & & & $(2,5)$ & $\leftrightarrow$ & 1 & 2 & . & 5 & \\
\hline 7 & -5 & -1 & -7 & 0 & -7 & $(4,3)$ & $\leftrightarrow$ & . & & 34 & . & \\
\hline 2 & -2 & -2 & -1 & -2 & 0 & $(2,1)$ & $\leftrightarrow$ & 1 & 2 & $\cdot$. & · & \\
\hline 5 & \begin{tabular}{|l|}
0 \\
\end{tabular} & -2 & -3 & -1 & -5 & $(5,4)$ & $\leftrightarrow$ & . & . & $\cdot 4$ & 5 & \\
\hline
\end{tabular}

Figure 5: Slacks at $\left(u_{x}, v_{x}\right)$ and the maximum excess intervals at $x$

is given. Since the intervals of maximum excess at $x$ form a completely separating family of coalitions, we have $s_{i j}(x)=-1=s_{j i}(x)$ for all $i \neq j$, so $x \in \mathcal{K}$.

Since there are two partitions among the maximal excess interval coalitions (corresponding to the off- $\pi$ entries having maximal slack of the two loops indicated in the slack matrix in Figure 5, one in darker gray), in procedure (2) we have $\varepsilon^{1}=-1$ and $\{[4,1],[2,3],[5,2],[3,4]\} \subseteq \Sigma_{1}$. Consequently, in this game $\mathcal{L C}=X^{1}$ is a lower dimensional subset of $\mathcal{C}=X^{0}$. The intervals in $\Sigma_{1}$ separate all pairs of players except 1 and 5 . It follows that any least-core allocation satisfies 9 of the $10=\left(\begin{array}{l}5 \\ 2\end{array}\right)$ kernel equalizers with surplus level -1 , hence, the kernel points are exactly those payoff vectors in $\mathcal{L C}=X^{1}$ for which $s_{15}()=.s_{51}($.$) also hold. Notice that the$ entire family of maximal excess intervals at $x$ is not balanced, the intervals $[1,2]$ and $[4,5]$ cannot be augmented with intervals from $\Sigma_{1}$ to form a balanced collection (accordingly, the corresponding off- $\pi$ entries $(2,1)$ and $(5,4)$ (in gray circles) are in no loop formed by maximal slack entries in the matrix).

It is easily checked that $\operatorname{dim}\left(X^{1}\right)=2$ (the rank of the membership vectors for coalitions in $\Sigma_{1}$ is 3 ) and $x$ is a vertex of $X^{1}$. However, from $x$ there is only one feasible direction in $X^{1}$ along which the payoff vectors also respect the $s_{15}()=.s_{51}($. kernel condition, namely $d^{1}=(-1,2,-2,2,-1)$. Since $\mathcal{K} \subseteq \mathcal{L C}=X^{1}$, around $x$ 
all kernel points are of the form $x+\lambda d^{1}$ for some $0 \leq \lambda \leq 1$. The situation at the other endpoint $y=x+d^{1}=(6,4,5,4,4)$ of this line segment is shown in Figure 6 with a particular choice of $\left(u_{y}, v_{y}\right) \in m^{-1}(y)$.

\begin{tabular}{c|ccccc|}
$u_{y} \backslash v_{y}$ & 1 & 2 & 0 & 2 & 0 \\
\cline { 2 - 5 } 5 & -5 & 0 & -5 & -1 & -5 \\
2 & -2 & -4 & 0 & -4 & -1 \\
\cline { 2 - 5 } 5 & -4 & -1 & -5 & 0 & -5 \\
2 & -3 & -4 & -1 & -4 & 0 \\
4 & 0 & -3 & -2 & -2 & -4 \\
\hline & & & & &
\end{tabular}

\begin{tabular}{|c|c|c|c|c|c|c|c|}
\hline$y$ & $=$ & 6 & 4 & & 4 & $4)$ & $\in X^{2} \subseteq \mathcal{K}$ \\
\hline$(1,4)$ & $\leftrightarrow$ & 1 & . & & 4 & 5 & \\
\hline$(3,2)$ & $\leftrightarrow$ & $\cdot$ & 2 & 3 & . & $\cdot$ & $\varepsilon^{1}=-1$ \\
\hline$(2,5)$ & $\leftrightarrow$ & 1 & 2 & & & 5 & \\
\hline$(4,3)$ & $\leftrightarrow$ & . & 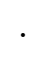 & 3 & 4 & . & \\
\hline$(2,1)$ & $\leftrightarrow$ & 1 & 2 & 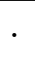 & . & . & \\
\hline$(5,3)$ & $\leftrightarrow$ & $\cdot$ & . & 3 & 4 & 5 & $\varepsilon^{2}=-2$ \\
\hline$(5,4)$ & $\leftrightarrow$ & . & . & & 4 & 5 & \\
\hline
\end{tabular}

Figure 6: Slacks at $\left(u_{y}, v_{y}\right)$ and intervals of the two highest excesses at $y$

Since there is a new partition among the coalitions having excess -2 , we get $\varepsilon^{2}=-2$ and $\{[1,2],[3,5]\} \subseteq \Sigma_{2}$ (the corresponding off- $\pi$ entries $(2,1)$ and $(5,3)$ with slack -2 form a new loop connected to one of the previous level loops). Since $[1,2]$ and $[3,5]$ separate 1 and 5 , it follows that $s_{15}(t)=-2=s_{51}(t)$ holds for any $t \in X^{2}$, so $X^{2} \subset \mathcal{K}$. The above allocation $x \in X^{1} \backslash X^{2}$ shows that the inclusion is indeed strict.

It is easily checked that $\operatorname{dim}\left(X^{2}\right)=1$ (the rank of the membership vectors for coalitions in $\Sigma_{1} \cup \Sigma_{2}$ is 4 ), so $X^{2}$ is a line segment with $y$ as one of its endpoints and $d^{2}=(-2,2,-2,2,0)$ as its directional vector from $y$. The other vertex of $X^{2}$ is $z=y+d^{2}=(4,6,3,6,4)$, as it is easily read from Figure 7 . Notice that the only change among the intervals having excess -2 at $y$ and at $z$ is that $[4,5]$ is replaced by its complement $[1,3]$ (the corresponding -2 slacks are in gray circles).

The nucleolus is the midpoint of $X^{2}$, where both $[4,5]$ and its complement $[1,3]$ have excess -3 (and all other not yet listed coalitions have excess not more than $-3)$, i.e., $\mathcal{N}=X^{3}=\left\{\frac{1}{2} y+\frac{1}{2} z\right\}=\{(5,5,4,5,4)\}$. On the other hand, the kernel is the union of the line segment $X^{2}$ and the line segment in $X^{1}$ joining $x$ and $y$, i.e.,

$$
\mathcal{K}=\{\lambda x+(1-\lambda) y: 0 \leq \lambda \leq 1\} \cup\{\lambda y+(1-\lambda) z: 0 \leq \lambda \leq 1\} .
$$

These two line segments have different directional vectors $\left(d^{1} \neq d^{2}\right)$, so the kernel is not convex. Indeed, at $w=\frac{1}{2} x+\frac{1}{2} z=(11 / 2,4,5,4,9 / 2)$ we have $s_{51}(w)=$ $e([3,5], w)=-5 / 2<-3 / 2=e([1,2], w)=s_{15}(w)$, so $w \in X^{1} \backslash \mathcal{K}$, implying that the kernel is not convex and it is indeed a proper subset of $X^{1}$. Therefore, in this 5 -player (cyclic) permutation game (which has a full-dimensional core) the claimed chain $\mathcal{C}=X^{0} \supset \mathcal{L C}=X^{1} \supset \mathcal{K} \supset X^{2} \supset \mathcal{N}$ of strict inclusions indeed holds. 


\begin{tabular}{c|ccccc|}
$u_{z} \backslash v_{z}$ & 1 & 4 & 0 & 4 & 0 \\
3 & -3 & 0 & -3 & -1 & -3 \\
2 & -2 & -6 & 0 & -6 & -1 \\
\cline { 2 - 5 } 3 & -2 & -1 & -3 & 0 & -3 \\
2 & -3 & -6 & -1 & -6 & 0 \\
4 & 0 & -5 & -2 & -4 & -4 \\
\hline & & & & &
\end{tabular}

\begin{tabular}{|c|c|c|c|c|c|c|c|}
\hline$z$ & $=$ & 4 & 6 & 3 & 6 & $4)$ & $\in X^{2} \subseteq \mathcal{K}$ \\
\hline$(1,4)$ & $\leftrightarrow$ & 1 & & & 4 & 5 & \\
\hline$(3,2)$ & $\leftrightarrow$ & $\cdot$ & 2 & 3 & $\cdot$ & . & $\varepsilon^{1}=-1$ \\
\hline$(2,5)$ & $\leftrightarrow$ & 1 & 2 & . & . & 5 & \\
\hline$(4,3)$ & $\leftrightarrow$ & . & & 3 & 4 & & \\
\hline$(2,1)$ & $\leftrightarrow$ & 1 & 2 & . & . & & \\
\hline$(5,3)$ & $\leftrightarrow$ & $\cdot$ & 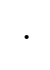 & 3 & 4 & 5 & $\varepsilon^{2}=-2$ \\
\hline$(3,1)$ & $\leftrightarrow$ & 1 & 2 & 3 & . & . & \\
\hline
\end{tabular}

Figure 7: Slacks at $\left(u_{z}, v_{z}\right)$ and intervals of the two highest excesses at $z$

\section{References}

[1] Curiel, I.J., Tijs, S.H. (1986) Assignment games and permutation games. Methods of Operations Research, 54:323-334.

[2] Davis, M., Maschler, M. (1965) The kernel of a cooperative game. Naval Research Logistics Quarterly, 12:223-259.

[3] Davis, M., Maschler, M. (1967) Existence of stable payoff configurations for cooperative games. In: Essays in Mathematical Economics in Honour of Oskar Morgenstern, M. Shubik, ed., Princeton University Press, Princeton, 39-52.

[4] Driessen, T.S.H. (1998) A note on the inclusion of the kernel in the core of bilateral assignment game. International Journal of Game Theory, 27:301-303.

[5] Granot, D. (1994) On a new bargaining set for cooperative games. Working paper, Faculty of Commerce and Business Administration, University of British Columbia, Vancouver, Canada.

[6] Granot D (2010) The reactive bargaining set for cooperative games. International Journal of Game Theory, 39:163-170.

[7] Granot, D., Granot, F. (1992) On some network flow games. Mathematics of Operations Research, 17:792-841.

[8] Maschler, M., Peleg, B., Shapley, L.S. (1972) The kernel and bargaining set for convex games. International Journal of Game Theory, 1:73-93.

[9] Maschler, M., Peleg, B., Shapley, L.S. (1979) Geometric properties of the kernel, nucleolus and related solution concepts. Mathematics of Operations Research, 4:303-338. 
[10] Meinhardt, H. I. (2014) The Pre-Kernel as a Tractable Solution for Cooperative Games: An Exercise in Algorithmic Game Theory. Theory and Decision Library C, Vol. 45, Springer, Berlin, 2014.

[11] Peleg B (1966) The kernel of the general-sum four-person game. Canadian Journal of Mathematics, 18:673-677.

[12] Peleg, B., Sudhölter, P. (2003) Introduction to the Theory of Cooperative Games. Kluwer Academic Publishers, Boston/Dordrecht/London, 2003.

[13] Quint, T. (1996) On one-sided versus two-sided matching games. Games and Economic Behavior, 16:124-134.

[14] Schmeidler, D. (1969) The nucleolus of a characteristic function game. SIAM Journal on Applied Mathematics, 17:1163-1170.

[15] Shapley, L.S. (1967) On balanced sets and cores. Naval Research Logistics Quarterly, 14:453-460.

[16] Shapley, L.S., Shubik, M. (1972) The assignment game I: The core. International Journal of Game Theory, 1:111-130.

[17] Solymosi, T. (2002) The bargaining set of four-person balanced games. International Journal of Game Theory, 31:1-11.

[18] Solymosi, T., Raghavan, T.E.S., Tijs, S. (2003) Bargaining sets and the core in permutation games. Central European Journal of Operations Research, 11:93101.

[19] Solymosi, T., Raghavan, T.E.S., Tijs, S. (2005) Computing the nucleolus of cyclic permutation games. European Journal of Operational Research, 162:270280 .

[20] Tijs, S.H., Parthasarathy, T., Potters, J.A.M., Rajendra Prasad, V. (1984) Permutation games: another class of totally balanced games. OR Spektrum, 6:119-123. 